\title{
Relación entre estrategias de historia de vida y atributos funcionales en especies arbóreas del Bosque Atlántico semideciduo de Misiones
}

\author{
Micaela Medina $^{1, \bigotimes ;}$; Luis J. Ritter ${ }^{2}$; Paula I. Campanello ${ }^{3} \&$ Marcelo F. Arturi $^{1}$ \\ ${ }^{1}$ Laboratorio de Investigación en Sistemas Ecológicos y Ambientales (LISEA), FCAyF, UNLP, La Plata, Argentina. ${ }^{2}$ Becario \\ CONICET, Facultad de Ciencias Forestales (UNaM). ${ }^{3}$ Centro de estudios ambientales integrados, Facultad de Ingeniería,
} UNPSJB, CONICET, Esquel, Argentina.

\begin{abstract}
Resumen. Caracterizar funcionalmente la respuesta de las plantas a factores ecológicos es un objetivo básico en ecología. Recientemente se describieron patrones globales de asociación entre atributos funcionales y estrategias de historia de vida, como especies adquisitivas vs. conservativas. En árboles tropicales se encontraron relaciones entre atributos morfológicos y reproductivos con diferentes requerimientos para la regeneración. En este trabajo se analizó si las clasificaciones ecológicas propuestas para especies de árboles frecuentes del bosque semideciduo de Misiones se relacionan con atributos funcionales obtenidos de bibliografía (densidad de madera [DM], altura de la planta $[\mathrm{H}]$, peso de semilla [PS] y atributos foliares), y también se evaluaron las relaciones bivariadas entre los atributos funcionales. Para ello se obtuvieron de la bibliografía la altura de la planta, la densidad de la madera, el peso de la semilla y el grupo ecológico de 62 especies de árboles frecuentes, y para 39 de esas mismas especies se recopiló el área foliar, el área foliar específica y el espesor de la hoja. Las especies clasificadas como pioneras tendieron a presentar menor densidad de la madera y menor altura que las clasificadas como climáxicas, pero esos valores se superpusieron en gran medida entre categorías intermedias. Esto sugiere que existe un gradiente funcional y remarca la dificultad de establecer categorías discretas. Las especies con mayor peso de semilla presentaron valores intermedios de densidad de madera. No hubo relación entre H, PS y DM y los atributos foliares, lo que sugiere que las características foliares representan un eje funcional independiente. Sobre la base de los resultados obtenidos y la información previa se propone un modelo conceptual gráfico para caracterizar a las especies del Bosque Atlántico de Misiones utilizando la densidad de la madera en relación con la tolerancia a la sombra, y la altura a la madurez como indicador de la estrategia de adquisición de luz y asociada con el síndrome de dispersión de las semillas.
\end{abstract}

[Palabras clave: clasificación funcional, densidad de la madera, altura, peso de semilla, bosques subtropicales]

\begin{abstract}
Aвstract. Relationship between life history strategies and functional traits in tree species in the Atlantic Forest in Misiones, Argentina. Functional characterization of plant responses to ecological factors is a basic goal in ecology. General trends were found indicating associations between functional traits and life history strategies such as acquisitive vs. conservative species. Different regeneration requirements among tropical trees were related to morphological and reproductive traits. Here, we studied if ecological groups of trees in the Atlantic Forest in Misiones are related to differences in height, wood density and seed mass. We also examined the relationships between those traits and leaf traits related to the acquisitive-conservative strategies. Plant height, wood density and seed mass were obtained from the literature for 62 common tree species in the studied area as well as their ecological classification. In addition, leaf area, specific leaf area and leaf thickness, were obtained from the literature for a subset of 39 tree species. Pioneer trees showed lower height and lighter wood than those classified as climax but these values widely overlapped among intermediate groups suggesting the existence of a functional gradient and that discrete classes are difficult to apply. Higher seed mass was observed among trees with medium wood density. No relationship between these traits and foliar traits was observed. Thus, the difference between species with acquisitive or conservative leaves constitutes a different functional axis from the one associated to wood density and height. Based on our results and previous findings we propose a conceptual model for the functional characterization of tree species in the Atlantic Forest in Misiones using wood density, as an indicator shade tolerance, and height, as an indicator of light acquisition strategy and related to dispersal syndrome of seeds.
\end{abstract}

[Keywords: functional classification, wood density, tree height, seed mass, subtropical trees]

Editora asociada: Natalia Pérez Harguindeguy ه mmedinamicaela88@gmail.com
Recibido: 28 de Septiembre de 2018

Aceptado: 31 de Julio de 2019 


\section{INTRODUCCIÓN}

Un objetivo básico de la ecología es caracterizar funcionalmente la respuesta de las plantas a factores ecológicos. La definición de estrategias de vida de plantas propone reconocer grupos de especies que se parecen por su respuesta frente a factores ecológicos (e.g., los disturbios, el estrés y la competencia [Grime1974]); consecuentemente, se establecen las especies ruderales, las competitivas y las tolerantes a la escasez de diferentes recursos. La historia de vida de los árboles tropicales recibió menos atención que la de los árboles templados y constituye un tema que aún se debate (Lugo and Zimmerman 2002; Nascimento et al. 2005). Tradicionalmente, las especies arbóreas tropicales fueron clasificadas por los requerimientos para su regeneración en especies que pueden regenerar bajo del dosel y especies que requieren aberturas del dosel para establecerse y crecer (Lugo and Zimmerman 2002). Esas tendencias fueron identificadas como un gradiente cuyos extremos son las especies intolerantes a la sombra (i.e., pioneras), en oposición a las tolerantes a la sombra (i.e., no pioneras) (Swaine and Whitmore 1988). Además, las diferencias en los requerimientos para su establecimiento fueron relacionadas con su respuesta a los disturbios y, en consecuencia, con tendencias sucesionales (Lugo and Zimmerman 2002). La categorización de las estrategias de historia de vida establecidas sobre la base de esos requerimientos abarca desde la simple definición de pioneras y no pioneras (Swaine and Whitmore 1988) hasta la diferenciación entre pioneras de vida corta, pioneras longevas y especies tardías discriminadas según su posición en el dosel (Lugo and Zimmerman 2002). En particular, en el Bosque Atlántico, diferentes estudios clasificaron a las especies en grupos ecológicos, denominados como categorías sucesionales, sobre la base de análisis de caracteres morfológicos (Souza et al. 2014), de observaciones de su regeneración a campo (Das Chagas e Silva and Soares-Silva 2000) o de conocimientos previos no sistematizados (Ferretti 1995; Carvalho et al. 2003). Estas clasificaciones utilizaron las categorías pioneras, secundarias iniciales, secundarias tardías y climáxicas, que representan una secuencia a lo largo de la cual tiende a disminuir la tasa de crecimiento y a aumentar la densidad de la madera (Das Chagas e Silva and Soares-Silva 2000; Souza et al. 2014). Además, estos autores indicaron que en esa misma secuencia aumenta el peso de la semilla, mientras que Souza et al. (2014) encontraron que el área foliar específica de las pioneras tendió a ser mayor que en las tolerantes a la sombra.

Las clasificaciones de las estrategias de historias de vida enfrentan el problema de utilizar categorías discretas para tratar un gradiente de respuestas de las especies frente a las condiciones heterogéneas que representa el bosque, desde un dosel continuo hasta diferentes tamaños de claros (Wright et al. 2003). Además, si bien muchos autores siguen utilizando estas clasificaciones, es frecuente que los criterios por los cuales una especie es incluida en un grupo determinado sean poco explícitos. El análisis de las relaciones entre clases discretas y caracteres cuantitativos permite entender los alcances y limitaciones del uso de categorías ecológicas discretas.

Una cantidad de trabajos describieron patrones globales de asociación entre atributos funcionales y estrategias de historia de vida de las plantas como las tasas demográficas, aptitud reproductiva y capacidad de las plantas para aprovechar recursos después de un disturbio (Díaz et al. 2016; Kunstler et al. 2016; Rüger et al. 2012; Westoby 1998). Por ejemplo, Westoby (1998) propuso que tres caracteres sencillos de medir (e.g., la altura de la planta, el peso de la semilla y el área foliar específica) pueden representar aspectos funcionales relacionados con las estrategias identificadas por Grime (1974). Sobre estas bases se caracterizó a las especies de crecimiento rápido, o llamadas también adquisitivas, frente a las de crecimiento lento, también conocidas comoconservativas (Wright et al. 2004; Adler et al. 2014; Reich 2014). Según esos trabajos, las primeras presentan menor densidad de madera, menor altura y mayor área foliar específica que las segundas. Se observó una tendencia en la que las hojas con menor tiempo de vida y mayor área foliar por unidad de masa tienden a tener tasas fotosintéticas más altas, lo cual corresponde a estrategias de rápida adquisición y consumo de recursos, en contraposición con las especies con hojas con mayor tiempo de vida y menor área foliar por unidad de masa, que presentan menores tasas fotosintéticas y corresponden a estrategias más conservativas (Wright et al. 2004). En especies arbóreas de bosque húmedos en Bolivia se encontró que las hojas con mayor área de la lámina correspondían a especies más demandantes de luz (Poorter and Bongers 2006), mientras que en especies arbóreas tropicales se encontró que el espesor 
de la hoja se correlacionó de forma positiva con las tasas fotosintéticas (Kenzo et al. 2006). Las estrategias adquisitivas y conservativas fueron asociadas, además, con caracteres del leño y, a su vez, se las vinculó con tasas de crecimiento y mortalidad (Adler et al. 2014; Reich 2014). En bosques tropicales de Panamá, Rüger et al. (2012) encontraron que tanto la densidad de la madera como el peso de la semilla se relacionan inversamente con las tasas de crecimiento. Las semillas livianas son producidas de manera abundante, presentan pocas reservas para nutrir a la plántula y corresponden a especies de rápido crecimiento que colonizan lugares desprovistos de vegetación (Grime 1974). Por el contrario, las especies con semillas pesadas, cuyas reservas son capaces de sostener a las plántulas en situaciones de escasez de recursos, corresponden a especies de bajo crecimiento como las que se establecen debajo del dosel. Estas relaciones son coherentes con los resultados de Adler et al. (2014), quienes encontraron tendencias globales que indican que tanto la fecundidad como la mortalidad son mayores en especies con menor peso de la semilla y menor densidad de la madera. También se reconoció a la altura de la planta a la madurez como un carácter importante para definir la historia de vida de los árboles tropicales (Swaine and Whitmore 1988), y se la consideró como un indicador de la longevidad (Worbes et al. 2003; Moles et al. 2009). Además, a la altura se la relacionó con la disponibilidad de luz debido a la posición que ocupan los individuos respecto del dosel, y también con un gradiente relacionado con el grado de recuperación de la vegetación después de un disturbio (Falster and Westoby 2005). Las especies más bajas son especies demandantes de luz que se establecen en etapas próximas a los disturbios, o bien especies tolerantes a la sombra que representan los estratos inferiores de bosques maduros. Las especies más altas, más allá de las condiciones en las que se establecen, caracterizan el dosel superior de bosques no afectados por disturbios recientes.

A su vez, entre las especies que forman parte de la estructura del dosel existe una amplia variación en sus estrategias de crecimiento (Brienen and Zuidema 2006). Mientras que algunas de ellas son especies demandantes de luz, rápido crecimiento y alta mortalidad bajo el dosel, otras son especies tolerantes a la sombra, de bajo crecimiento y que se establecen bajo el dosel (Brienen and Zuidema 2006). Estudios realizados en el Bosque
Atlántico indicaron que caracteres foliares como la densidad de la madera pueden contribuir a identificar diferentes grados de tolerancia a la sombra (Campanello et al. 2011) y tendencias sucesionales (Vicente Silva et al. 2016). Sin embargo, otros trabajos indicaron la inexistencia de asociación entre esos caracteres y el crecimiento en especies arbóreas subtropicales, lo cual indica que tales relaciones podrían depender de condiciones locales (Li et al. 2017).

ElBosque Atlántico semideciduodeMisiones, Paraguay y Sur de Brasil se encuentra entre los sistemas boscosos más diversos del mundo, y también entre los más amenazados (Di Bitetti et al. 2003). En consecuencia, resulta clave entender las relaciones entre los atributos funcionales de las especies arbóreas y su respuesta frente a diferentes disturbios, su adaptación a diferentes formas de manejo del bosque, tal que resulten útiles para implementar medidas eficientes de restauración o rehabilitación ecológica. Este trabajo tiene como objetivo general comparar categorías discretas utilizadas para analizar grupos ecológicos de árboles con un abordaje cuantitativo de atributos funcionales. En particular, este trabajo tiene tres objetivos específicos: 1) comparar formalmente si la clasificación ecológica en pioneras, secundarias iniciales, secundarias tardías y climáxicas, propuesta para especies de árboles del Bosque Atlántico de Misiones, se relacionan con atributos funcionales como la densidad de la madera - como indicador de la tasa de crecimiento y de la demanda de luz-, la altura de la planta — como indicador de su estrategia de captación de luz y de su longevidad-, y el peso de la semilla - como un indicador de la estrategia reproductiva y dispersiva -; 2) analizar las relaciones entre densidad de la madera, altura de la planta y peso de semilla, y la relación de estas variables con características de las hojas (área foliar específica y el espesor de la lámina), y 3) proponer un modelo conceptual, basado en atributos de fácil estimación, que permita interpretar diferencias en las estrategias de historia de vida de las especies arbóreas del Bosque Atlántico semideciduo de Misiones.

\section{Materiales y MÉTOdos}

\section{Área de estudio}

Se incluyeron las especies arbóreas comúnmente citadas en la ecorregión Selva 
Paranaense. La Selva Paranaense pertenece al complejo de ecorregiones conocido como Bosque Atlántico y abarca el SE de Brasil, Paraguay y NE de la Argentina (Oyarzabal et al. 2018), en particular llamadas Bosque Atlántico del Alto Paraná (Di Bitetti et al. 2003) y Bosque mixto con Araucaria (Araucaria angustifolia [Bertol] Kuntze). La vegetación predominante fue considerada como bosque húmedo semideciduo con unas 200 especies arbóreas que conforman tres estratos (Rodríguez and Silva 2012). La temperatura media anual en la región varía entre $15^{\circ} \mathrm{C}$ y 23 ${ }^{\circ} \mathrm{C}$, y la precipitación anual entre 1000 y 2000 mm (Rodríguez and Silva 2012).

\section{Caracteres funcionales y grupos ecológicos}

Para determinar si la especie presentaba hábito arbóreo se siguió la base de datos del Instituto de Botánica Darwinion. Se utilizó la nomenclatura botánica de la misma base de datos. Se trabajó con una lista de 62 especies, para las que se dispuso de la densidad de la madera (DM) (Mainieri and Peres-Chimelo 1989; Atencia 2003; Zanne et al. 2010; Rodríguez 2015), la altura de la planta (H) (López et al. 1987; Ortega Torres et al. 1989) y el peso de la semilla (PS) (Carvalho 2003). Se calculó el promedio en aquellas especies que en diferentes fuentes indicaron distintos valores. En el caso de la altura de la planta, el valor consignado en la mayoría de los casos representa un valor aproximado de la estatura de la planta a la madurez (López et al. 1987). Además, para 39 de esas 62 especies se dispuso de valores de área foliar (AF), área foliar específica (AFE) y el espesor de la hoja (EH) (Prado et al. 2015; Silva 2015; Nascimento 2017; Rodrigues et al. 2018). Todos los trabajos citados determinaron los atributos foliares siguiendo el protocolo descrito por Cornelissen et al. (2003). La lista de especies con sus respectivos nombres científicos, familia, grupo ecológico y valores de los atributos se adjunta en los Apéndices 1 y 2. Por otro lado, se utilizaron cuatro variables para identificar a los cuatro grupos ecológicos considerados: pioneras, secundarias iniciales, secundarias tardías y climáxicas, indicando con " 1 " al grupo o grupos a los que fue asignada cada especie y " 0 " en otro caso. Estos grupos fueron propuestos en los trabajos de Ferretti (1995); Das Chagas e Silva and SoaresSilva (2000) y Carvalho (2003). Las fuentes de variación quedaron incluidas en los análisis aquí realizados.
Se evaluaron las tendencias de variación de la densidad de la madera, la altura de la planta y el peso de la semilla entre los distintos grupos ecológicos mediante gráficos de caja. Sin embargo, no se realizaron pruebas formales para evaluar las diferencias entre grupos debido a que la inclusión de especies en más de un grupo afectó la independencia de los datos. Con el objetivo de evaluar las relaciones de asociación entre los atributos funcionales considerados se llevaron a cabo gráficos de dispersión y se calcularon coeficientes de correlación por rangos de Spearman, con un nivel de significancia de 5\%. Las relaciones multivariadas entre estos atributos funcionales se analizaron mediante análisis de componentes principales (ACP).

Se realizaron pruebas de Mantel (Legendre and Fortin 1989) con el fin de analizar la relación entre la clasificación ecológica y los atributos funcionales. A partir de la lista de 62 especies se calculó una matriz de atributos funcionales por especie (DM, PS y H) y otra de cuatro variables indicando los grupos ecológicos por especie (pionera, secundaria inicial, secundaria tardía y climáxica). Las pruebas de Mantel se llevaron a cabo aplicando una estandarización a los valores de cada atributo consistente en la sustracción de la media y la división por el desvío estándar. Esta estandarización es requerida para datos medidos en diferentes unidades y con varianzas diferentes (Oksanen et al. 2013). A partir de la lista de 39 especies para las cuales se contó con atributos foliares (AFE, AF y EH) se aplicaron pruebas de Mantel para evaluar si las distancias entre especies calculadas sobre la base de la altura, la densidad de madera y el peso de la semilla se correlacionaban con las distancias entre especies calculadas sobre la base de caracteres foliares. Las correlaciones de Mantel y el ACP se llevaron a cabo con el paquete Vegan (Oksanen et al. 2013) del programa de uso libre $\mathrm{R}$ ( $\mathrm{R}$ Development Core Team 2014).

\section{Resultados}

De las 62 especies se registraron 12 clasificadas como pioneras, 8 como climáxicas, 33 como secundarias iniciales y 36 como secundarias tardías. Sin embargo, 18 especies fueron identificadas como secundarias iniciales y tardías, lo que indica que más de la mitad de las especies de esos grupos fueron compartidas (18 de 33 y 18 de 38). 
Las especies pioneras tendieron a presentar menor densidad de la madera y peso de semilla que las especies climáxicas, pero no se observaron tendencias claras en las categorías
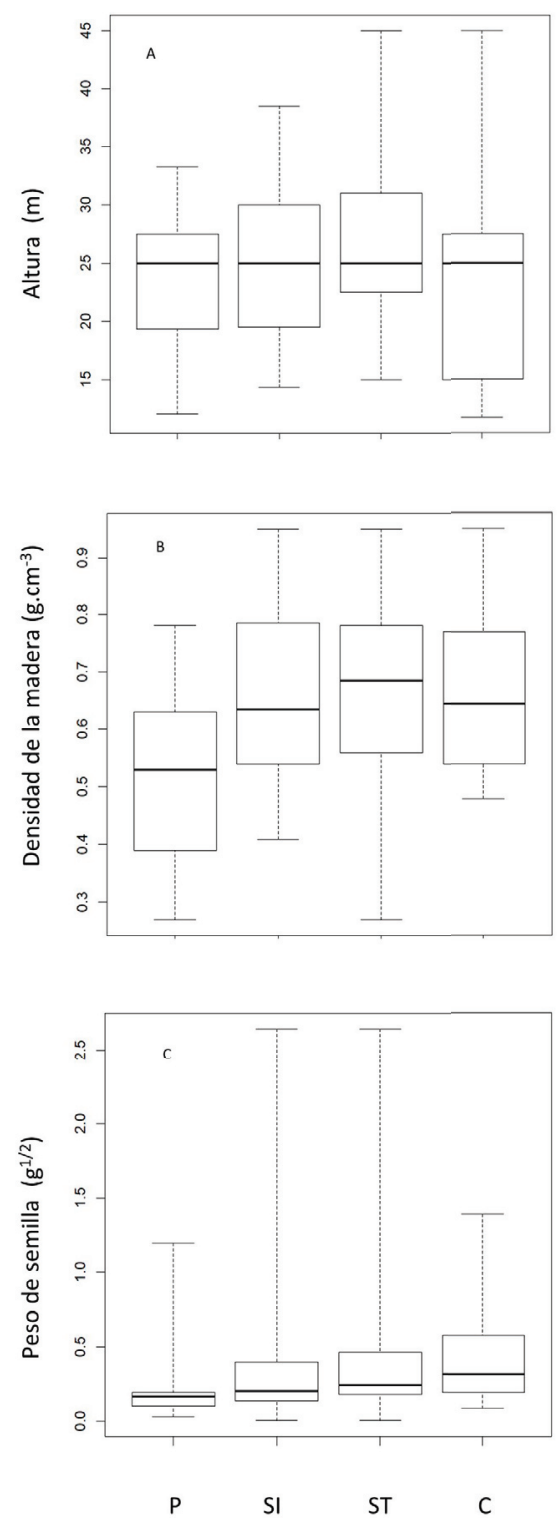

Figura 1. Caracteres funcionales por grupos ecológicos para 62 especies arbóreas del Bosque Atlántico Semideciduo en Misiones, Argentina. El peso de la semilla se expresa como raíz cuadrada para mejorar la visualización. P: pioneras; SI: secundarias iniciales; ST: secundarias tardías; C: climáxicas. A) Altura vs. grupos ecológicos. B) Densidad de madera vs. grupos ecológicos. C) Peso de semilla vs. grupos ecológicos.

Figure 1. Functional traits per ecological group for 62 tree species from Semideciduous Atlantic Forest in Misiones, Argentina. Seed mass is expressed as squared root for better visualization. P: pioneers; SI: initial secondaries; ST: late secondaries; C: climax. A) Height vs. ecological group. B) Wood density vs. ecological group. C) Seed mass vs. ecological group. intermedias (Figura 1). La altura a la madurez tendió a aumentar desde las pioneras hacia las climáxicas. El análisis de mantel indicó una correspondencia significativa entre las clasificaciones ecológicas y las distancias entre especies calculadas por la altura de la planta, la densidad de la madera y el peso de la semilla ( $\mathrm{r}=0.17 ; \mathrm{P}=0.014)$, aunque esta relación sólo se observó al remover Aracauria angustifolia (Bertol) Kuntze (araucaria) del análisis debido a la alta influencia del peso de la semilla de esta especie. Por el contrario, la prueba de Mantel no arrojó resultados significativos al comparar a las especies por la altura a la madurez, la densidad de la madera y el peso de la semilla con los caracteres foliares $(r=0.09$, $\mathrm{P}>0.32)$.

Las asociaciones bivariadas entre la altura, la densidad de la madera y el peso de la semilla no resultaron significativas (Figura 2). El peso de la semilla tendió a presentar sus valores más altos y mayor variabilidad para valores intermedios de la densidad de la madera, aunque araucaria representó un valor atípico en esa tendencia por el alto peso de su semilla. Por su parte, el área foliar mostró una relación negativa con el aumento de la densidad de la madera, y el espesor de la hoja tuvo sus valores más altos para las especies de menor densidad de la madera (Figura 3). Los restantes caracteres foliares fueron independientes de la densidad de la madera y la altura a la madurez.

El eje 1 del ACP (42.3\% de varianza explicada) se asoció de manera positiva con la densidad de la madera (carga en el autovector $=0.71$ ) (Figura 4). Hacia el extremo negativo se localizaron especies con baja densidad de la madera como Cecropia pachystachya Trécul. (ambay), Solanum granulosum-leprosum Dunal (fumo bravo) y Trema micrantha (L.) Blume, (palo pólvora). El eje 2 (32.7\% de la varianza explicada) se asoció de forma positiva con el peso de la semilla (carga en el autovector $=0.65$ ) y negativa con la altura de la planta a la madurez (carga en el autovector=-0.76). Hacia el extremo positivo del eje 2 se ubicaron las especies con mayor peso de semilla y menor altura, mientras que en el lado negativo de este eje se ubicaron las especies con características opuestas como Apuleia leiocarpa (vogel) J. F. Macbr (grapia), Aspidosperma polyneuron Müll. Arg. (palo rosa) y Peltophorum dubium (Spreng.) Taub. (caña fístula). 

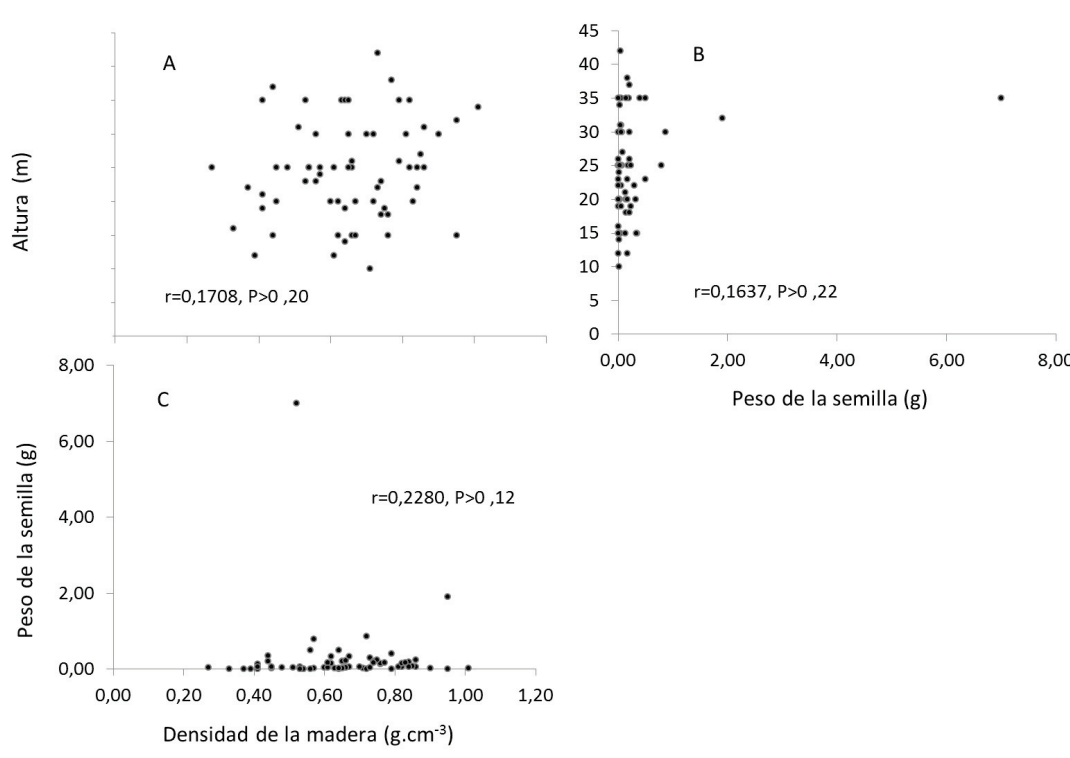

Figura 2. Relaciones bivariadas entre la altura a la madurez, la densidad de la madera y el peso promedio de la semilla para 62 especies del Bosque Atlántico Semideciduo de Misiones, Argentina. R: coeficiente de correlación de Spearman; P: valor de probabilidad. A) Altura vs. densidad de madera. B) Altura vs. peso de semilla. C) Peso de semilla vs. densidad de madera.

Figure 2. Bivariate relationships between height atmaturity, wood density and seed mass for 62 trees species form the Semideciduous Atlantic Forest in Misiones, Argentina. R: Spearman correlation coefficient; P: probability value. A) Height vs. wood density. B) Height vs. Seed mass. C) Seed mass vs. wood density.
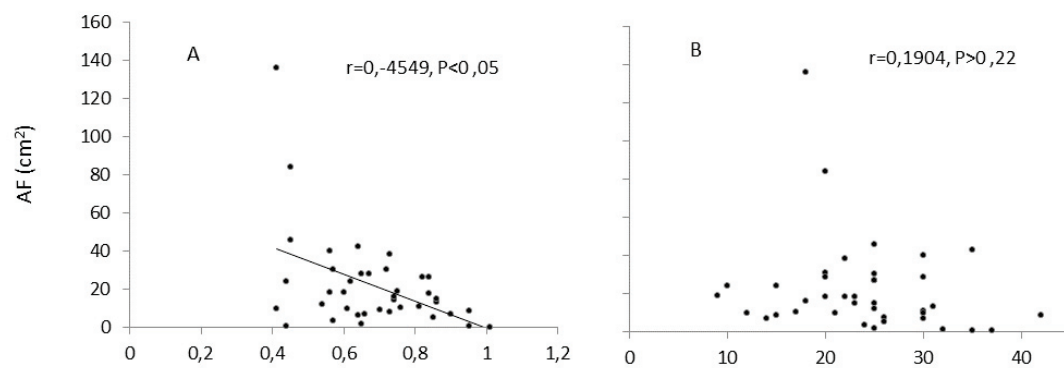

Figura 3. Relaciones bivariadas entre caracteres foliares y la densidad de la madera y la altura a la madurez para 39 especies del Bosque Atlántico de Misiones. R: coeficiente de correlación de Spearman; P: valor de probabilidad del estadístico. A)Área foliar vs densidad de madera
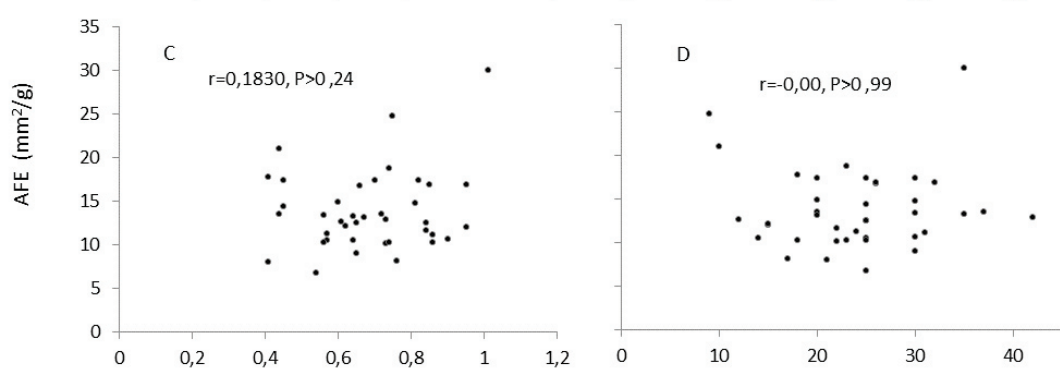

B) Area foliar vs altura

C) Área foliar específica vs densidad de madera D)Área foliar específica vs altura E) Espesor de la hoja vs densidad de madera F) Espesor de la hoja vs altura.

Figure 3. Bivariate relationships between wood density, height at maturity and leaf traits for 39 trees species form the
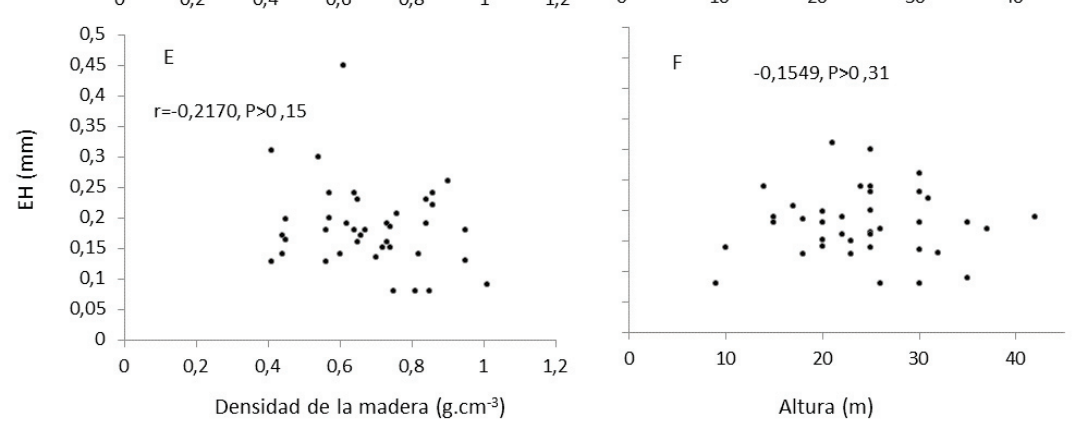
Semideciduous Atlantic Forestin Misiones, Argentina. r: Spearman correlation coefficient, P: probability value.A) Leaf area vs wood density B) Leaf area vs height C) Specific leaf area vs wood density D) Specific leaf area vs height E) Leaf thickness vs wood density F) Leaf thickness vs height 

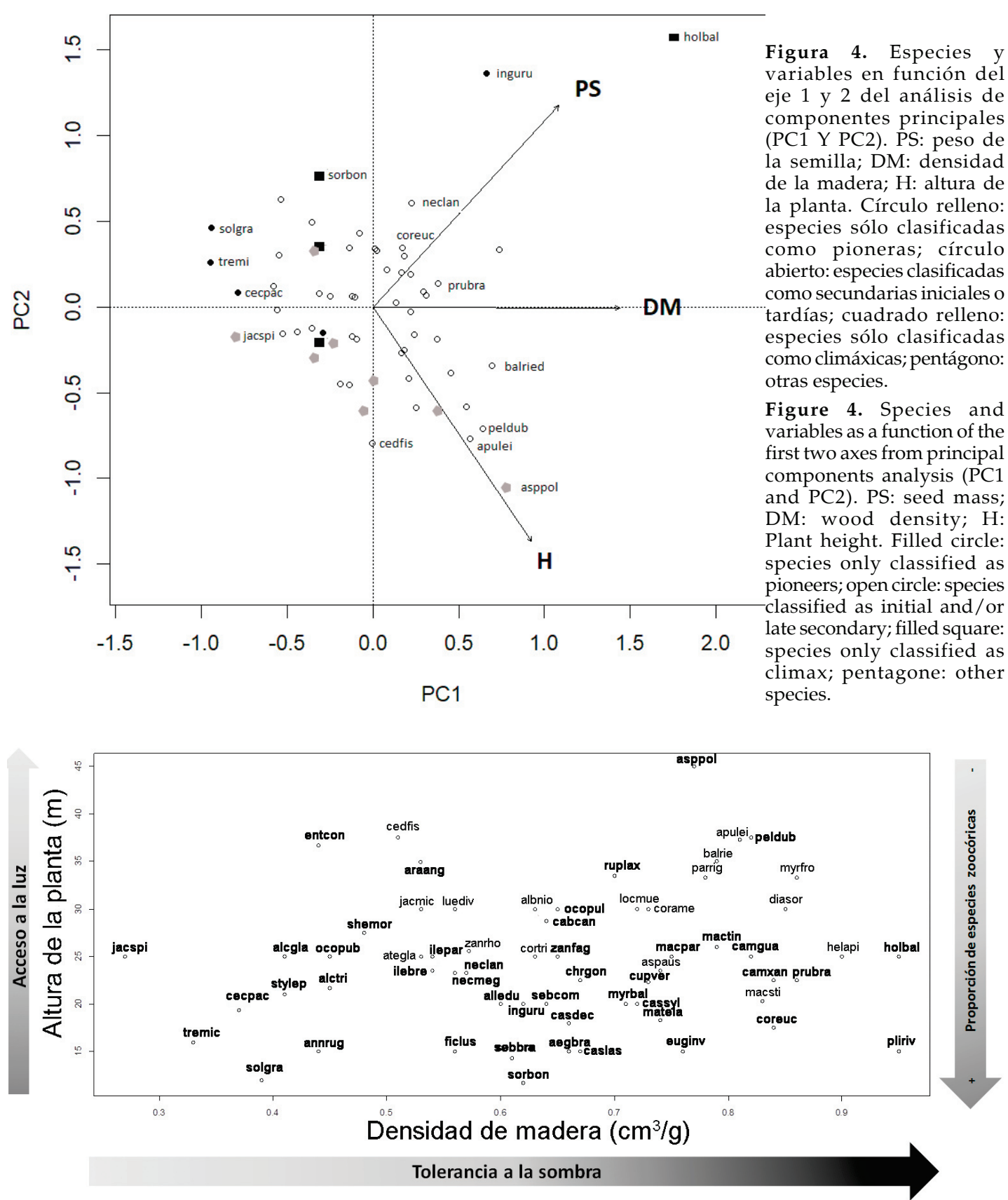

Figura 5. Modelo conceptual basado en la densidad de madera y la altura a la madurez para 62 especies arbóreas estudiadas del Bosque Atlántico Semideciduo de Misiones, Argentina. Las especies que están en negrita presentan dispersión zoocórica.

Figure 5. Conceptual model based on wood density and height at maturity traits for 62 trees species form the Semi deciduous Atlantic Forest in Misiones, Argentina. Zoochoric species are in bold

\section{DISCUSIÓN}

Los grupos ecológicos utilizados por diferentes autores para diferenciar estrategias de historias de vida se corresponden con diferencias en la densidad de la madera, el peso de la semilla y la altura de la planta a la madurez. Las diferencias más claras se observaron entre especies clasificadas como pioneras y las clasificadas como climáxicas. Sin embargo, las categorías intermedias presentaron valores muy similares entre 
ellas en todas las variables analizadas, lo que indica que representan un gradiente continuo de respuestas ecológicas y que podrían presentar mayor plasticidad. Este resultado concuerda con lo hallado por Wright et al. (2003), quienes proponen que existe un amplio rango de variación en los requerimientos de las especies que se encuentran entre los dos extremos representados por especies pioneras y no pioneras propuesto por Swaine and Whitmore (1988). Además, Welden et al. (1991) indicaron que más del $70 \%$ de las especies de árboles tropicales pueden tolerar niveles bajos de radiación.

No se encontraron relaciones entre los caracteres foliares y la densidad de la madera o la combinación de los tres caracteres (altura de la planta, densidad de la madera y peso de la semilla). Heberling y Friedley (2012) indicaron que las tendencias de asociación de caracteres presentadas por Wright et al. (2004) como el espectro de economía de las hojas podría variar al ser analizado localmente debido a que los ensambles de especies pueden responder a compromisos en el uso de diferentes recursos como luz y agua o estar influenciados por relaciones filogenéticas que condicionan las correlaciones entre caracteres ( $\mathrm{Du}$ and Mao 2015). No esta claro si las especies de árboles se comportan siguiendo una única estrategia en relación a la asignación de recursos al tallo y las hojas (i.e., si las especies que tienen alta densidad de madera tienen también hojas resistentes). En un trabajo en el que se estudiaron 668 especies de árboles de bosques amazónicos se observó que las características relacionadas con los costos de construcción de los tallos (e.g., densidad de madera, grosor de corteza) y de las hojas son independientes y, por lo tanto, una especie que tiene densidad de madera alta no necesariamente tiene hojas más resistentes (Baraloto et al. 2010). La existencia de compromisos funcionales complejos en relación con el uso de luz y el agua podría resultar esperable en el Bosque Atlántico semideciduo porque en esta ecorregión conviven especies de bosques secos y húmedos (Oyarzabal et al. 2018). Además, las relaciones entre los caracteres analizados podrían verse afectadas por el uso de valores obtenidos de la bibliografía y sujetos a variación ambiental, geográfica y metodológica; en consecuencia, resulta recomendabledisponer deestimaciones locales de los caracteres (Wright et al. 2010).

El peso de la semilla tendió a coincidir con lo esperado para un gradiente de tolerancia a la sombra. Diversos trabajos establecieron que las semillas livianas son producidas abundantemente, presentan pocas reservas capaces de nutrir a la plántula y corresponden a especies de rápido crecimiento que colonizan lugares sin vegetación (Grime 1974). Por el contrario, las especies con semillas pesadas, cuyas reservas son capaces de sostener a las plántulas en situaciones de escasez de recursos, corresponden a especies de bajo crecimiento como las que se establecen debajo del dosel. Rüger et al. (2012) encontraron en bosques tropicales de Panamá que tanto la densidad de la madera como el peso de la semilla se relacionan inversamente con las tasas de crecimiento. Sumado a esto, para el set de especies analizadas, el peso de semilla puede estar relacionado con la altura en relación a la dispersión de las mismas. Excepto por pocas especies (araucaria y Holocalyx balansae Micheli [alecrín]), las especies del dosel superior tienden a presentar semillas livianas, dispersadas por el viento, mientras que las del dosel intermedio y bajo tienden a presentar mayor peso de semilla y son dispersadas por animales. Esa relación fue independiente de la densidad de la madera.

El uso de la densidad de la madera para caracterizar la historia de vida de árboles tropicales y subtropicales resulta más confiable que otros caracteres, ya que la relación inversa entre la densidad de la madera, el crecimiento y la demanda de luz representa un resultado consistente tanto en el Bosque Atlántico de Misiones (Campanello et al. 2011) como en bosques amazónicos (Nascimento et al. 2005) y en bosques tropicales en Panamá (Wright et al. 2003; Rüger et al. 2012). Sobre la base de esas relaciones y de los resultados obtenidos en este trabajo se propone un modelo conceptual gráfico para caracterizar las especies del bosque Atlántico de Misiones (Figura 5). En el modelo, la densidad de la madera se relaciona de forma positiva con la tolerancia a la sombra, mientras que la altura a la madurez se relaciona positivamente con el acceso a la luz. Además, la altura, podría relacionarse con el modo de la dispersión de las semillas, ya que la mayoría de las especies menores a 30 $\mathrm{m}$ de altura son zoocóricas y las mayores de $30 \mathrm{~m}$ tienden a ser anemócoras o autócoras. Las especies con madera de baja densidad y una altura a la madurez inferior a $15 \mathrm{~m}$ son especies con alta demanda de luz (intolerantes a la sombra), vida corta y crecimiento rápido. Por ejemplo, palo pólvora es frecuente luego de un disturbio que ocasiona la reducción del dosel (Vázquez-Yanes 1998). Otras especies 
similares, pero más longevas y menos demandantes de luz, están representadas por ambay y Alchornea triplinervia (Spreng.) Müll. Arg. (mora blanca). Por otro lado, especies con mayor densidad de la madera, alturas menores de $20 \mathrm{~m}$ a la madurez, caracterizan el estrato arbóreo inferior del bosque maduro, como Allophyllus edulis (A. St.-Hil, A. Juss. and Cambess) Hieron. Ex Niederl. (cocú), Sorocea bonplandi (Baill.) W. C. Burger, Lanj. and Wess. Boer (ñandipá), Plinia rivularis (Cambess.) Rotman. (guporetí) y Eugenia spp., entre otras. Entre las especies que pueden alcanzar el dosel superior se observa un gradiente de densidad de madera que podría reflejar diferencias en los requerimientos para establecerse y crecer. En un extremo se encuentran Enterolobium contortisiliqulum (Vell.) Morong. (timbó colorado) y Bastardiopsis densiflora (Hook. and Arn.) Hassl. (loro blanco), que presentan baja densidad de la madera y se establecen en sitios con baja cobertura del dosel (aunque su crecimiento aumenta con mayor disponibilidad luz). En el otro extremo se encuentran las especies del dosel superior, con mayor densidad de madera y baja demanda de luz (tolerantes a la sombra), que pueden establecerse en bosques con dosel continuo y alcanzar el dosel con crecimiento más lento como Holocalyx balansae Micheli (alecrín). Situaciones intermedias de especies del dosel superior podrían estar representadas por Cedrella fissilis Vell. (cedro misionero) y Cabralea canjerana (Vell.) Mart (cancharana).
Estas especies presentan densidades intermedias de madera y plasticidad necesaria para desarrollar tasas de crecimiento altas con niveles de radiación elevados, y sobrevivir bajo el dosel siempre que la radiación no disminuya a valores muy bajos (Campanello et al. 2008; Moretti et al. 2019).

En conclusión, al comparar especies pioneras con climáxicas, los grupos ecológicos existentes se relacionan con la densidad de la madera y con el peso de la semilla; sin embargo, existe confusión entre las categorías intermedias, que probablemente representan un gradiente en los requerimientos para la regeneración. La diferenciación entre especies sobre la base de la altura, de la densidad de la madera y del peso de la semilla no se relacionó con los caracteres foliares, lo que sugiere que, entre las especies arbóreas estudiadas, esos caracteres representan ejes funcionales independientes. La caracterización de las especies del Bosque Atlántico Semideciduo de Misiones sobre la base de la densidad de la madera y la altura a la madurez permite diferenciar con facilidad estrategias de historia de vida con objetivos de manejo y rehabilitación ecológica.

Agradecimientos. Este trabajo contó con la financiación parcialdela Universidad Nacional de La Plata, el Proyecto Plantaciones Forestales Sustentables, Unidad de Cambio Rural, Ministerio de Agroindustria de la Nación y el Consejo Nacional de Investigaciones Científicas y Tecnológicas.

\section{REFERENCIAS}

Adler, P. B., R. Salguero - Gómez, A. Compagnoni, J. S. Hsu, J. Ray - Mukherjee, C. Mbeau - Ache, and M. Franco. 2014. Functional traits explain variation in plant life history strategies. PNAS 111(2):740-745. https://doi.org/10.1073/ pnas.1315179111.

Atencia, M. E. 2003. Densidad de maderas. Argentina: INTI - CITEMA.

Baraloto, C., T. Paine, L. Poorter, J. Beauchene, D. Bonal, A. M. Domenach, B. He'rault, S. Patin, J. C. Roggy, and J. Chave. 2010. Decoupled leaf and stem economics in rain forest trees. Ecology Letters 13:1338-1347. https://doi.org/ 10.1111/j.1461-0248.2010.01517.x.

Brienen, R. W., and P. A. Zuidema. 2006. Lifetime growth patterns and ages of Bolivian rain forest trees obtained by tree ring analysis. Journal of Ecology 94:481-493. https:/ / doi.org/10.1111/j.1365-2745.2005.01080.x.

Campanello, P. I., G. Gatti, and G. Goldstein. 2008. Coordination between water-transport efficiency and photosynthetic capacity in canopy tree species at different growth irradiances. Tree Physiology 28:85-94. https://doi.org/10.1093/ treephys/28.1.85.

Campanello, P. I., M. G. Gatti, L. Montti, M. Villagra, and G. Goldstein. 2011. Ser o no ser tolerante a la sombra: economía de agua y carbono en especies arbóreas del Bosque Atlántico (Misiones, Argentina). Ecología Austral 21:285-285.

Carvalho, P. E. R. 2003. Espécies Arbóreas Brasileiras. Brasília. Brasil. Empresa Brasileira de Pesquisa Agropecuária (EMBRAPA). EMBRAPA Florestas. Pp. 1039.

Cornelissen, J. H. C., S. Lavorel, E. Garnier, S. Díaz, N. Buchmann, D. E. Gurvich, P. B. Reich, H. ter Steege, H. D. Morgan, M. G. A. van der Heijden, J. G. Pausas, and H. Poorter. 2003. A handbook of protocols for standardized and easy measurement of plant functional traits worldwide. Australian Journal of Botany 51:335-380. https://doi.org/ 10.1071/BT02124.

Das Chagas e Silva, F., and L. H. Soares-Silva. 2000. Arboreal flora of the Godoy forest statepark, Londrina, Pr. Brazil. Edinburgh Journal of Botany 57:107-120. https:/ / doi.org/10.1017/S096042860000007X.

Díaz, S., and M. Cabido. 2001. Vive la Difference: Plant Functional Diversity Matters to Ecosystem Processes. Trends 
in Ecology and Evolution 16:646-655. https://doi.org/10.1016/S0169-5347(01)02283-2.

Díaz, S., J. Kattge, J. H. C. Cornelissen, I J. Wright, S. Lavorel, et al. 2016. The global spectrum of plant form and function. Nature 529(7585):167-171. https://doi.org/10.1038/nature16489. https://doi.org/10.1038/nature16489.

Di Bitetti, M., G. Placci, and L. Dietz. 2003. Una visión de biodiversidad para la ecoregión del Bosque Atlántico del Alto Paraná: Diseño de un Paisaje para la Conservación de la Biodiversidad y prioridades para las acciones de conservación. Washington, D.C., USA. World Wildlife Fund. Pp. 156.

Falster, D. S., and M. Westoby. 2005. Alternative height strategies among 45 dicot rain forest species from tropical Queensland, Australia. Journal of Ecology 93(3):521-535. https://doi.org/10.1111/j.0022-0477.2005.00992.x.

Ferretti, A. R., P. Y. Kageyama, G. F. Árboez, J. D. Santos, M. I. A. Barros, R. F. Lorza, and C. Oliveira. 1995. Classificação das espécies arbóreas em grupos ecológicos para revegetação com nativas no estado de São Paulo. Florestar Estatístico 3(7):73-77.

Forgiarini, C., A. F. Souza, S. J. Longhi, and J. M. Oliveira. 2015. In the lack of extreme pioneers: trait relationships and ecological strategies of 66 subtropical tree species. Journal of Plant Ecology 8(4):359-367. https://doi.org/10.1093/ jpe/rtu028.

Grime, J. P. 1974. Vegetation classification by reference to strategies. Nature 250:26-31. https://doi.org/10.1038/ $250026 \mathrm{a} 0$.

Heberling, J. M., and J. D. Fridley. 2012. Biogeographic constraints on the world-wide leaf economics spectrum. Global Ecology and Biogeography 21:1137-1146. https://doi.org/10.1111/j.1466-8238.2012.00761.x.

Kenzo, T., T. Ichie, Y. Watanabe, R. Yoneda, I. Ninomiya, and T. Koike. 2006. Changes in photosynthesis and leaf characteristics with tree height in five dipterocarp species in a tropical rain forest. Tree Physiology 26:865-873. https: //doi.org/10.1093/treephys/26.7.865.

King, D. A, S. J. Davies, M. N. Nur Supardi, and S. Tan. 2005. Tree growth is related to light interception and wood density in two mixed dipterocarp forests of Malaysia. Functional Ecology 19:445-453. https://doi.org/10.1111/j.13652435.2005.00982.x.

Kunstler, G., D. Falster, D. A. Coomes, F. Hui, R. M. Kooyman, et al. 2016. Plant functional traits have globally consistent effects on competition. Nature 529(7585):204-207. https://doi.org/10.1038/nature16476.

Lavorel, S., and E. Garnier. 2002. Predicting changes in community composition and ecosystem functioning from plant traits-revisiting the Holy Grail. Functional Ecology 16:545-556. https:// doi.org/10.1046/j.1365-2435.2002.00664.x.

Li, Y., W. Kröber, H. Bruelheide, W. Härdtle, and G. von Oheimb. 2017. Crown and leaf traits as predictors of subtropical tree sapling growth rates. Journal of Plant Ecology 10:136-145. https://doi.org/10.1093/jpe/rtw041.

Legendre, P., and M. J. Fortin. 1989. Spatial pattern and ecological analysis. Vegetatio 80:107-138. https://doi.org/ 10.1007/BF00048036.

López, J. A., E. L. Little Jr., G. F. Ritz, J. S. Rombold, and W. J. Hahn. 1987. Árboles comunes del Paraguay. Ñandeyvyra mata kuera, Cuerpo de Paz, Asunción, Paraguay.

Lugo, A. E., and J. K. Zimmerman. 2002. Ecological Life Histories. Pp. 191-213 en J. A. Vozzo (ed.). Tropical seed manual. Agricultural Handbook 721. Washington DC USDA Forest Service.

Mainieri, C., and J. Peres-Chimelo. 1989. Fichas de características das maderas brasileiras. Instituto de Pesquisas Tecnológicas. Sao Paulo, Brasil. Pp. 129-130.

Moles, A. T., D. I. Warton, L. Warman, N. G. Swenson, S. W. Laffan, A. E. Zanne, A. Pitman, F. A. Hemmings, and M. R. Leishman. 2008. Global Patterns in plant height. Journal of Ecology 97:923-932. https://doi.org/10.1111/j.13652745.2009.01526.x.

Moretti, A. P., F. Y. Olguin, M. A. Pinazo, F. Gortari, J. Vera Bahima, and C. Graciano. 2019. Supervivencia y crecimiento de un árbol nativo maderable bajo diferentes coberturas de dosel en el Bosque Atlántico, Misiones, Argentina. Ecología Austral 29:099-111. https://doi.org/10.25260/EA.19.29.1.0.779.

Nascimento, H. E. M., W. Laurance, R. Condit, S. Laurance, S. D'Angelo, and A. C. Andrade. 2005. Demographic and life-history correlates for Amazonian trees. Journal of Vegetation Science 16:625-634. https:/ /doi.org/10.1111/j.16541103.2005.tb02405.x.

Nascimento, D. R. 2017. Artificial reservoirs affect tree functional components of tropical dry forests. Pós-Graduação em Ecologia Aplicada ao Manejo e Conservação de Recursos Naturais. Universidade Federal de Juiz de Fora, Brazil. Pp. 36.

Ortega Torres, E., L. Stutz de Ortega, R. Spichiger. 1989. Flora del Paraguay. Conservatoire et Jardinbotaniques de la Ville de Geneve. Pp. 217.

Poorter, L., and F. Bongers. 2006. Leaf traits are good predictors of plant performance across 53 rain forest species. Ecology 87:1733-1743. https://doi.org/10.1890/0012-9658(2006)87[1733:LTAGPO]2.0.CO;2.

Prado Júnior, J., I. Schiavini, V. Vale, S. Lopes, C. Arantes, and A. P. Oliveira. 2015. Functional leaf traits of understory species: strategies to different disturbance severities. Brazilian Journal Biology 75:339-346. https:/ / doi.org/10.1590/ 1519-6984.12413.

Reich, P. B. 2014. The world"wide 'fast-slow' plant economics spectrum: a traits manifesto. Journal of Ecology 102: 275-301. https://doi.org/10.1111/1365-2745.12211.

Rodríguez, A., and M. Silva. 2012. Ecorregión Selva Paranaense. Pp. 719 en J. Morello, S. Mateucci, A. Rodríguez and M. Silva (eds.). Ecorregiones y complejos ecosistémicos argentinos. Orientación Gráfica Editora. Buenos Aires. MONOGRAF 1a. ed. XXXII.

Rodrigues, A. V., F. L. V. Bones, A. Schneiders, L. Z. Oliveira, A. C. Vibrans, and A. L. Gasper. 2018. Plant Trait Dataset 
for Tree-Like Growth Forms of the Subtropical Atlantic Rain Forest (Version 1.1). Data 3(2). https:/ / doi.org/10.3390/ data3020016.

Rodríguez, S. A. 2015. Propiedades biomecánicas y Propiedades biomecánicas y ecofisiológicas de especies de ecofisiológicas de especies de árboles nativos y cultivados en la provincia de Misiones. Tesis Doctoral Facultad de Ciencias Exactas y Naturales Universidad de Buenos Aires, Buenos Aires, Argentina.

Rüger, N., C. Wirth, S. J. Wright, and R. Condit. 2012. Functional traits explain light and size response of growth rates in tropical tree species. Ecology 93:2626-2636.

Rüger, N., L. S., Comita, R. Condit, D. Purves, B. Rosenbaum, M. D. Visser, S. J. Wright, and C. Wirth. 2018. Beyond the fast-slow continuum: demographic dimensions structuring a tropical tree community. Ecology Letters 21:10751084. https://doi.org/10.1890/12-0622.1.

Silva, M. G. 2015. Padrões funcionais de comunidades de plantas lenhosas em transições floresta-campo em resposta a gradientes ambientais no sul do Brasil. Mestre em Ecología. Universidade Federal do Rio Grande do Sul, Porto Alegre. Brasil. Pp. 76.

Souza, A. F., C. Forgiarini, S. J. Longhic, and D. A. Brena. 2008. Regeneration patterns of a long-lived dominant conifer and the effects of logging in southern South America. Acta Oecologica 34:221-232. https:/ /doi.org/10.1016/ j.actao.2008.05.013.

Souza, A. F, C. Forgiarini, S. J. Longhi, and J. Morales Oliveira. 2014. Detecting ecological groups from traits: a classification of subtropical tree species based on ecological strategies. Brazilian Journal of Botany 37:441-452. https: //doi.org/10.1007/s40415-014-0084-z.

Swaine, M. D., and T. C. Whitmore. 1988. On the definition of ecological species groups in tropical rain forests. Vegetatio 75:81-86. https://doi.org/10.1007/BF00044629.

Vázquez-Yanes, C. 1998. Trema micrantha (L.) Blume (Ulmaceae): A promising neotropical tree for site amelioration of deforested land. Agroforestry Systems 40(1):97-104. https:/ / doi.org/10.1023/ A:1006063010677.

Vicente-Silva, J., R. S. Bergamin, K. J. Zanini, V. D. Pillar, and S. C. Müller. 2016. Assembly patterns and functional diversity of tree species in a successional gradient of Araucaria forest in Southern Brazil. Natureza and Conservação 14:67-73. https://doi.org/10.1016/j.ncon.2016.09.006.

Westoby, M. 1998. A leaf-height-seed (LHS) plant ecology strategy scheme. Plant and Soil 199:213-227. https:/ /doi.org/ 10.1023/A:1004327224729.

Worbes, M., R. Stachel, A. Roloff, and W. J. Junk. 2003. Tree ring analysis reveals age structure, dynamics and wood production of a natural forest stand in Cameroon. Forest Ecology and Management 173:105-123. https://doi.org/ 10.1016/S0378-1127(01)00814-3.

Wright, I. J., P. B. Reich, M. Westoby, D. D. Ackerly, Z. Baruch, et al. 2004. The worldwide leaf economics spectrum. Nature 428:821-827. https://doi.org/10.1038/nature02403.

Wright, S. J., H. C. Muller-landau, R. Condit, and S. P. Hubbell. 2003. Gap-dependent recruitment, realized vital rates, and size distributions of tropical trees. Ecology 84:3174-3185. https://doi.org/10.1890/02-0038.

Wright, S. J., K. Kitajima, N. J. B. Kraft, P. B. Reich, I. J. Wright, D. E. Bunker, R. Condit, J. W. Dalling, S. J. Davies, S. Díaz, B. M. J. Engelbrecht, K. E. Harms, S. P. Hubbell, C. O. Marks, M. C. Ruiz-Jaen, and C. M. Salvador. 2010. Functional traits and the growth-mortality trade" off in tropical trees. Ecology 12:3664-3674. https://doi.org/10.1890/09-2335.1.

Zanne, A. E., M. Westoby, D. S. Falster, D. D. Ackerly, S. R. Loarie, S. E. J. Arnold, and D. A. Coomes. 2010. Angiosperm wood structure: global patterns in vessel anatomy and their relation to wood density and potential conductivity. American Journal of Botany 97(2):207-215. https://doi.org/10.3732/ajb.0900178. 